\title{
miR-188 suppresses tumor progression by targeting SOX4 in pediatric osteosarcoma
}

\author{
LU PAN $^{1}$, LINGXIN MENG $^{2}$, FENG LIANG $^{1}$ and $\mathrm{LICAO}^{3}$ \\ Departments of ${ }^{1}$ Pediatric Surgery, ${ }^{2}$ Oncology and ${ }^{3}$ Anaesthesia Operation, \\ People's Hospital of Rizhao, Rizhao, Shandong 276800, P.R. China
}

Received January 23, 2018; Accepted April 17, 2018

DOI: $10.3892 / \mathrm{mmr} .2018 .8997$

\begin{abstract}
RNA-188 (miR-188) acts as a tumor suppressor in various types of human cancer, including glioma, oral squamous cell carcinoma and hepatocellular carcinoma. However, the function and mechanism of miR-188 in pediatric osteosarcoma (OS) have yet to be investigated. In the present study reverse transcription-quantitative polymerase chain reaction revealed that miR-188 expression was significantly downregulated in pediatric OS tissues and cell lines. miR-188 overexpression markedly suppressed OS cell proliferation, migration and invasion, and induced cellular apoptosis. An in vivo assay demonstrated that miR-188 overexpression inhibited tumor growth. miR-188 targeted SOX4 to regulate its expression. miR-188 expression was inversely correlated with SOX4 in pediatric OS tissues. SOX4 restoration abrogated the inhibitory effects of miR-188 on OS cells. The results of the present study indicated that miR-188 suppressed pediatric OS progression by targeting SOX4.
\end{abstract}

\section{Introduction}

Osteosarcoma (OS) is the most aggressive and widespread bone tumor that typically occurs in children and adolescents (1). OS causes numerous cancer-related deaths in adolescents and children every year because of rapid progression, high metastasis ability, and poor clinical outcomes $(2,3)$. At present, OS is mainly treated with surgical resection combined with standard chemotherapy and radiation (4). Although considerable efforts have been devoted to developing novel effective methods for OS treatment, minor advances have been achieved, and the 5-year overall survival rate of patients with OS remains low (5). Therefore, the pathogenesis of OS must be determined.

Correspondence to: Professor Lu Pan, Department of Pediatric Surgery, People's Hospital of Rizhao, 126 Tai'an Road, Xinshi, Rizhao, Shandong 276800, P.R. China

E-mail:panlu2018@163.com

Key words: microRNA-188, SOX4, progression, pediatric osteosarcoma
MicroRNAs (miRNAs) are a class of noncoding RNAs that measure a length of 18-25 nucleotides and reportedly participate in nearly all kinds of biological processes (6-9). miRNAs play essential roles in human cancers, such as head and neck carcinomas (10), hepatocellular carcinoma (11), colon cancer (12), lung cancer (13), and OS (14), by regulating cellular proliferation, migration, invasion, and apoptosis. miRNAs can target the 3'-UTR of specific mRNAs and regulate gene expression. The dysregulation of miRNA expression results in the development or progression of cancers (15). For example, miRNA-140 suppresses OS tumor growth by enhancing the anti-tumor immune response (16). miRNA-665 suppresses the invasion and metastasis of OS by directly inhibiting RAB23 (17). miR-188 is implicated in the regulation of tumor occurrence. For instance, Zhang et al (18) reported that miR-188-5p inhibits tumor growth and metastasis in prostate cancer by repressing LAPTM4B expression. However, the role of miR-188 in OS remains largely unknown.

In the present study, we demonstrated that miR-188 was downregulated in OS tissues compared with that in adjacent normal tissues. miR-188 overexpression inhibited the proliferation, migration, and invasion of OS cells by directly targeting SOX4. Our findings indicated that miR-188 acted as a tumor suppressor and might be a promising target for OS treatment.

\section{Materials and methods}

Clinical specimens and cell lines. Seventy-four pediatric patients with OS (age range: 5-21 years) were recruited from the People's Hospital of Rizhao, and adjacent normal tissues were obtained from the same specimens. Tumor biopsies were collected prior to neoadjuvant therapy, immediately frozen, stored at $-80^{\circ} \mathrm{C}$, and histologically characterized by a pathologist. This study was approved by the Ethics Committee of the People's Hospital of Rizhao and all patients gave written informed consent for the usage of their tissues within the present study.

The OS cell lines U2OS, SAOS2, and MG63 and the fetal osteoblastic cell line hFOB1.19 were purchased from the American Type Culture Collection (Manassas, VA, USA) and cultured in DMEM at $37^{\circ} \mathrm{C}$ in a humidified incubator with $5 \% \mathrm{CO}_{2}$. 
Oligonucleotide and transfection. miR-188 mimics and miR-control were chemically synthesized by GenePharma (Shanghai, China). The cells were transfected with miR-188 mimics or miR control by using Lipofectamine reagent (Invitrogen; Thermo Fisher Scientific, Inc., Waltham, MA, USA). The cells were harvested for further experiments $24 \mathrm{~h}$ after transfection.

Cell proliferation assay. Cell viability was monitored by Cell Counting Kit (CCK)-8 (Dojindo Molecular Technologies, Inc., Kumamoto, Japan). $5 \times 10^{3}$ OS cells were seeded onto 96-well plates and incubated for 1, 2 and 3 days. Subsequently, $10 \mu 1$ of CCK8 reagents were added to the 96-well plates, after $2 \mathrm{~h}$ incubation at $37^{\circ} \mathrm{C}$, the absorbance at $450 \mathrm{~nm}$ was measured to evaluate the number of viable cells by SUNRISE microplate reader (Tecan Group, Ltd., Mannedorf, Switzerland).

In vitro migration and invasion assays. The transwell assay was conducted in 24-well BD Matrigel invasion chambers (BD Biosciences, Franklin Lakes, NJ, USA) according to the manufacturer's instructions. Briefly, $5 \times 10^{4}$ OS cells were seeded in the upper well of the migration chamber in DMEM without serum, and $500 \mu 1$ DMEM supplemented with $10 \%$ FBS were added to the lower chamber well. After $24 \mathrm{~h}$ incubation, the cells on the top of the well were removed with a cotton swab, and the bottom cells were fixed with $4 \%$ paraformaldehyde, subsequently stained with $0.1 \%$ crystal violet for $30 \mathrm{~min}$. Images were captured in 5 independent fields. For invasion assay, the membranes were coated with Matrigel (BD Biosciences).

Reverse transcription-quantitative polymerase chain reaction. Total RNA was extracted from cultured cells using TRIzol reagent (Invitrogen; Thermo Fisher Scientific, Inc.) according to the manufacturer's protocol and cDNA was synthesized from total RNA by a PrimerScript RT Reagent kit (Takara Bio, Inc., Otsu, Japan). MiRNA from total RNA was reverse transcribed using the Prime-Script miRNA cDNA Synthesis kit (Takara Bio, Inc.). Real-time PCR (RT-PCR) was performed with the SYBR-Green Premix Ex Taq II (Takara Bio, Inc.) on Applied Biosystems Step One Plus Real-Time PCR System (Applied Biosystems; Thermo Fisher Scientific, Inc.). GAPDH was used as the endogenous control for detection of mRNA expression level, while U6 was used as endogenous control for miRNA expression analysis. Relative gene expression level was calculated by $2^{-\Delta \Delta \mathrm{CT}}$ method (19).

Tumor xenograft model. Eight female BALB/c nude mice aged 4-6 weeks were used for the tumor growth assay. U2OS cells $\left(3 \times 10^{6}\right)$ transfected with miR-188 or control were subcutaneously injected into the dorsal flank of the nude mice. Tumor volume was measured at indicated time points. The mice were sacrificed on the 4 th week to evaluate the tumor growth. All animal experiments were performed with the approval of the People's Hospital of Rizhao.

RNA immunoprecipitation (RIP). The RIP experiment was performed using the Magna RIP ${ }^{\mathrm{TM}}$ RNA-Binding protein immunoprecipitation kit (EMD Millipore, Billerica, MA, USA) following manufacturer's protocol. U2OS and SAOS2 cells were lysed using complete RIP lysis bufferand $100 \mu \mathrm{l}$ of the whole cell extract was incubated with RIPA buffer containing magnetic beads conjugated with human anti-Argonaute2 (Ago2) antibody (EMD Millipore) for 6-8 h at $4^{\circ} \mathrm{C}$. Normal mouse IgG (EMD Millipore) was used as a negative control. Samples were washed with washing buffer and incubated with proteinase $\mathrm{K}$ at $55^{\circ} \mathrm{C}$ for $30 \mathrm{~min}$ to isolate the RNA-protein complexes from beads. Then immunoprecipitated RNA was extracted and subjected to qRT-PCR analysis.

Luciferase reporter assay. MiR-188 mimic, pRL-CMV Renilla luciferase reporter was co-transfected into the cells using Lipofectamine 2000 (Invitrogen; Thermo Fisher Scientific, Inc.). After 48 h, luciferase data was measured by using a luciferase assay kit (Promega Corporation, Madison, WI, USA). Firefly luciferase activity was normalized against Renilla luciferase activity.

Statistical analysis. All statistical analyses were performed using SPSS v20.0 (IBM Corp., Armonk, NY, USA) and GraphPad Prism. Student's t-test and one-way ANOVA followed by Tukey's post hoc test were used to analyze 2 or multiple groups, respectively, for statistical significance. Pearson correlation coefficient analysis was used to determine the correlations. $\mathrm{P}<0.05$ was considered to indicate a statistically significant difference.

\section{Results}

miR-188 was overexpressed in OS tissues and cell lines. To investigate the role of miR-188 in the regulation of OS progression, we initially analyzed the expression of miR-188 in 74 pairs of OS tissues and adjacent normal tissues through real-time quantitative polymerase chain reaction (RT-qPCR). The results indicated that the miR-188 expression was downregulated in tumor tissues compared with that in normal tissues (Fig. 1A). We then determined the expression patterns of miR-188 in OS cell lines by RT-qPCR. We found that miR-188 was underexpressed in OS cell lines, including U2OS, MG63, and SAOS2 cells (Fig. 1B). We also analyzed the correlation of miR-188 expression with the clinical characteristics of OS tissues and found that the miR-188 expression level was negatively correlated with the tumor size, metastasis, and TNM stage of OS (Table I).

Upregulation of miR-188 inhibited OS cell proliferation but induced apoptosis. To investigate the function of miR-188 in OS cells, we overexpressed miR-188 in U2OS and SAOS2 cells by transfecting them with miR-188 mimics. RT-qPCR revealed that the miR-188 expression was significantly upregulated in U2OS and SAOS2 cells (Fig. 2A). We then performed CCK8 assays to assess the effects of miR-188 on OS cell proliferation. The miR-188 overexpression significantly inhibited the proliferation of U2OS and SAOS2 cells (Fig. 2B and C). We analyzed the cell cycle by FACS and observed that miR-188 overexpression markedly inhibited the cells in the $\mathrm{S}$ phase (Fig. 2D), indicating that miR-188 inhibited cell cycle progression. Moreover, AnnexinV/PI staining showed that miR-188 overexpression significantly increased the apoptotic U2OS and SAOS2 cells (Fig. 2E). To further evaluate the effects of 
Table I. Correlation between clinicopathological features and the expression of miR-188 in pediatric OS tissues.

\begin{tabular}{lccc}
\hline & \multicolumn{2}{c}{ miR-188 } & \\
\cline { 2 - 3 } Feature & Low & High & P-value \\
\hline All cases & 40 & 34 & \\
Tumor size (cm) & & & 0.035 \\
$<5$ & 14 & 21 & \\
$\geq 5$ & 26 & 13 & \\
Metastases & & & 0.034 \\
No & 13 & 20 & \\
Yes & 27 & 14 & 0.012 \\
Clinical stage & & & \\
I/II & 15 & 23 & \\
III & 25 & 11 & \\
\end{tabular}

The median expression level was used as the cutoff.
A

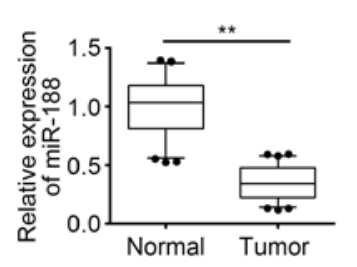

B

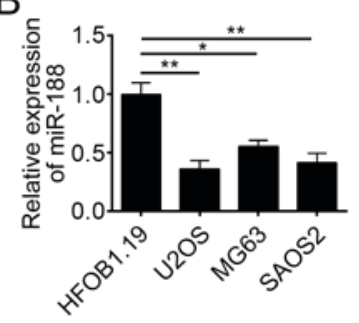

Figure 1. miR-188 overexpression in OS tissues and cell lines. (A) The fold change of miR-188 expression in human OS tissues and adjacent normal tissues was measured by reverse transcription-quantitative polymerase chain reaction. (B) The relative expression level of miR-188 in human OS cell lines (U2OS, SAOS2, and MG 63) and fetal osteoblastic cell line (hFOB). Data are representative of three independent experiments and expressed as the mean \pm standard deviation. ${ }^{*} \mathrm{P}<0.05$ and ${ }^{* *} \mathrm{P}<0.01$. OS, osteosarcoma; miR, microRNA.

miR-188 on tumor growth, we conducted a xenograft experiment and measured the tumor volumes at the indicated time points. We found that miR-188 overexpression significantly delayed the tumor growth in vivo (Fig. $2 \mathrm{~F}$ ). We evaluated the tumor weights at the end of the experiment and demonstrated that miR-188 overexpression significantly reduced the tumor size (Fig. 2G). Overall, miR-188 overexpression prevented OS cell proliferation but induced apoptosis.

MiR-188 overexpression inhibited OS cell migration and invasion. Tumor metastasis substantially contributed to the poor outcomes of patients with OS. To assess the influence of miR-188 on tumor metastasis, we conducted Transwell assays. miR-188 overexpression significantly inhibited the migration and invasion of U2OS and SAOS2 cells in vitro (Fig. 3A and B).

SOX4 is a direct target of miR-188 in OS cells. miRNAs regulate the expression of target genes. In our study, we investigated the miR-188-mediated molecular mechanism of
OS. We predicted the target gene of miR-188 by using the TargetScan and PicTar algorithm software and found that SOX4 is a potential target of miR-188. SOX4 is a key oncogene in OS development $(20,21)$. Therefore, we chose SOX4 for further investigation. As shown in Fig. 4A, an associating site of miR-188 possibly existed in the 3'-UTR of the mRNA of SOX4. It is well documented that miRNAs are presented in the cytoplasm in the form of miRNA ribonucleoprotein complexes (miRNPs) containing Ago2, a key component of RNA-induced silencing complex (RISC). Therefore, RIP assay was conducted in U2OS and SAOS2 cells using antibody Ago2 to verify whether SOX4 and miR-188 were in the same RISC complex. The RIP assay results demonstrated that SOX4 and miR-188 were both significantly enriched in Ago2-containing miRNPs relative to control group (Fig. 4B), suggesting that SOX4 and miR-188 were in the same RISC complex. To further explore whether SOX4 could directly interact with miR-188, luciferase reporter plasmids containing the wild-type or mutated miR-188 binding sites in SOX4 3'-UTR were constructed, as presented in Fig. 4A, and cotransfected with miR-control or miR-188 into U2OS and SAOS2 cells. Luciferase reporter assay showed that ectopic expression of miR-188 significantly reduced the luciferase activity of SOX4 3'-UTR-WT but not that of SOX4 3'-UTR-Mut (Fig. 4C). Taken together, these data indicated that SOX4 directly interacted with miR-188. Furthermore, we found that miR-188 overexpression significantly inhibited the mRNA and protein levels of SOX4 in U2OS and SAOS2 cells (Fig. 4D and E). Finally, the expression of SOX4 was inversely correlated with that of miR-188 in OS tissues (Fig. 4F). Overall, our findings demonstrated that SOX4 is a target of miR-188 in OS cells.

Ectopic SOX4 expression alleviated the miR-188-mediated inhibition of the proliferation, migration, and invasion of $O S$ cells. We analyzed the expression patterns of SOX4 in OS cells through RT-qPCR. SOX4 expression was upregulated in OS cell lines compared with that in hFOB cells (Fig. 4A). The SOX4 expression was upregulated in OS tissues compared with that in normal tissues (Fig. 5B). To determine whether SOX4 is responsible for the inhibitory function of miR-188 in OS cells, we restored the SOX4 expression in miR-188-overexpressed U2OS and SAOS2 cells (Fig. 5C). Subsequent functional analyses demonstrated that the restoration of SOX4 rescued the miR-188-mediated reduction of the proliferation, migration, and invasion of U2OS and SAOS2 cells but inhibited their apoptosis (Fig. 5D-G). Therefore, miR-188 prevented OS progression by inhibiting SOX4.

\section{Discussion}

In the present study, we investigated the role of miR-188 in OS cells. miR-188 expression was downregulated in OS tissues compared with that in adjacent normal tissues. The ectopic miR-188 expression suppressed the proliferation, migration, and invasion of OS cells but promoted the apoptosis of these cells. We also identified SOX4 as a direct target gene of miR-188 in OS cells. miR-188 and SOX4 expression were negatively correlated in OS tissues. The restoration of SOX4 could also reverse the miR-188-mediated effect on OS cells. 

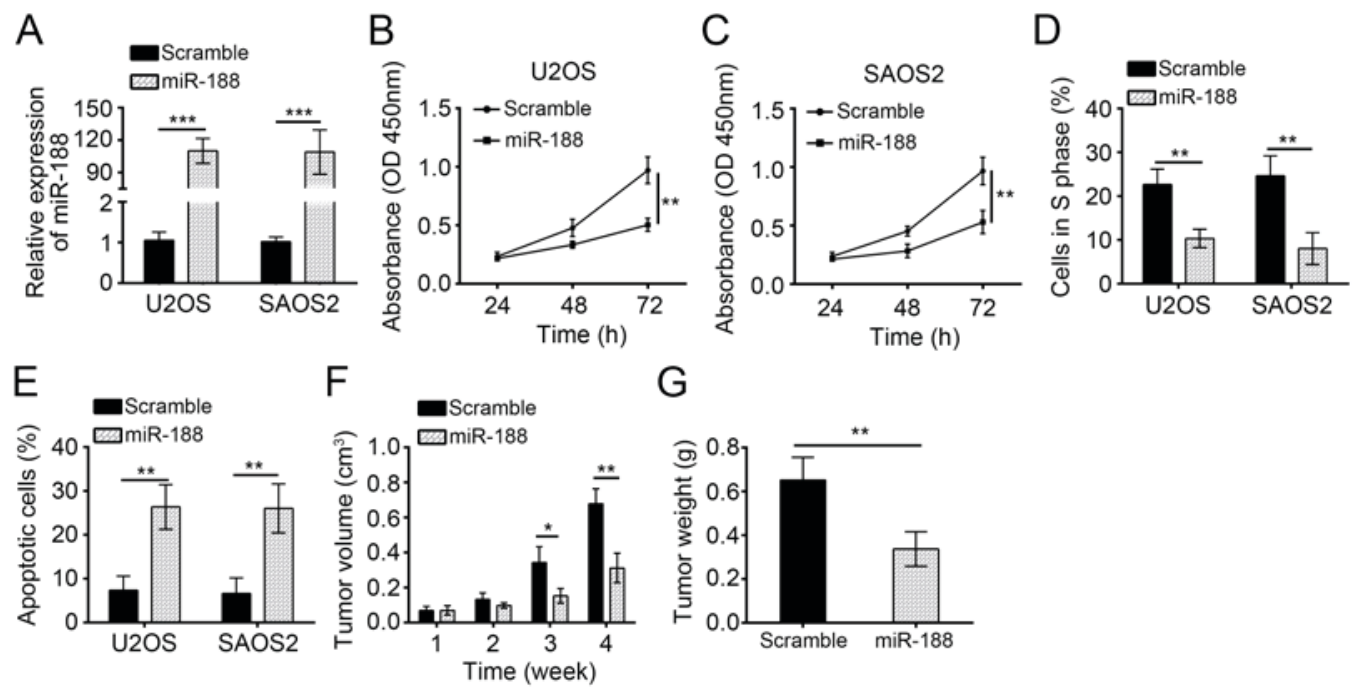

G

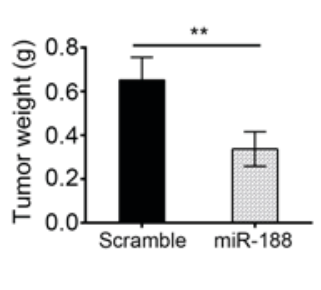

Figure 2. miR-188 upregulation inhibits OS cell proliferation and induces apoptosis. (A) miR-188 overexpression significantly increased the relative miR-188 expression level in U2OS and SAOS2 cells. A Cell counting kit-8 assay indicated that miR-188 overexpression reduced the proliferation of (B) U2OS and (C) SAOS2 cells. (D) A fluorescence activated cell sorter assay revealed that miR-188 overexpression inhibited cell entry into the S phase. (E) miR-188 overexpression increased the ratio of apoptotic cells. Cells were stained with AnnexinV/propidium iodide. (F) Tumor volume was determined at the indicated time points. (G) Tumor weight was measured at the end of the experiment. Data are representative of three independent experiments and expressed as mean \pm standard deviation. ${ }^{*} \mathrm{P}<0.05,{ }^{* *} \mathrm{P}<0.01$ and ${ }^{* * *} \mathrm{P}<0.001$. OD, optical density; OS, osteosarcoma; miR, microRNA.
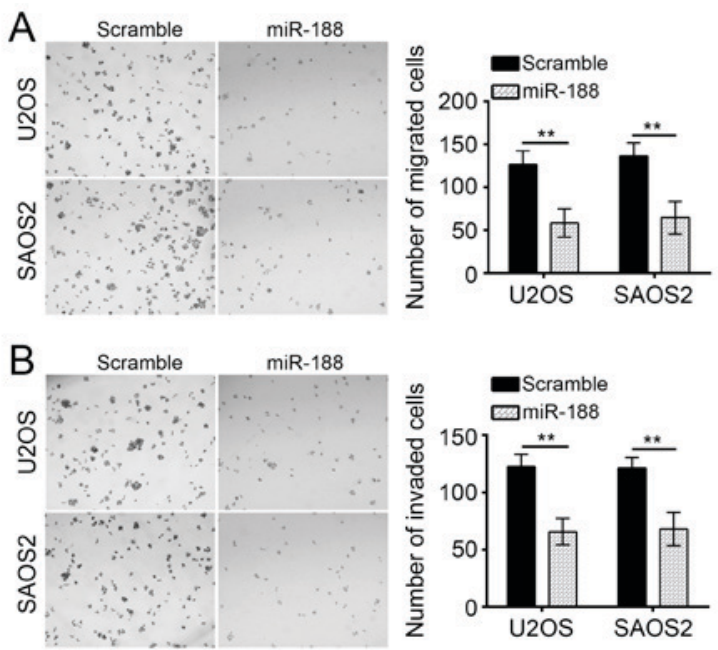

Figure 3. miR-188 overexpression inhibits OS cell migration and invasion miR-188 overexpression suppressed OS cell (A) migration and (B) invasion. Magnification, $x 20$. Data are representative of three independent experiments and expressed as mean \pm standard deviation. ${ }^{* *} \mathrm{P}<0.01$. OS, osteosarcoma; miR, microRNA.

Therefore, miR-188 served as a tumor suppressor in the development and progression of OS.

miRNAs are a class of noncoding RNAs that have a length of 18-25 nucleotides and modulate gene expression via posttranscriptional regulation (22-24). miRNAs are important regulators for OS development and progression. For instance, Tian et al (25) reported that the miR-635 expression is significantly decreased in OS specimens, and miR-635 overexpression inhibits OS development by enhancing cell apoptosis. Jia et al (26) showed that miR-300 decreases the cell viability, inhibits the migration, and promotes the apoptosis of OS cells by downregulating Twist1. In addition, miR-302a suppresses the proliferation, migration, and invasion of OS cells by targeting ADAM9 (27). Previous studies reported that miR-188 acts as a tumor suppressor in certain cancers. For example, Wang et al (28) reported that miRNA-188 is downregulated in oral squamous cell carcinoma, and it inhibits proliferation and invasion by targeting SIX1. However, the functions of miR-188 in OS have yet to be defined. In our study, we confirmed that miR-188 overexpression significantly inhibited OS growth in vitro and in vivo. By contrast, the ectopic miR-188 expression significantly enhanced cellular apoptosis. The miR-188 expression in OS tissues was positively correlated with tumor size, metastasis, and TNM stage. Therefore, we demonstrated the tumor suppressive role of miR-188 in OS.

SOX4 is a member of the Sry-related high-mobility group box (Sox) family of transcription factors and closely related to the development and progression of various cancers (29). An abnormal SOX4 overexpression is often linked to tumorigenicity and cancer stemness (30). SOX4 is upregulated in various cancers, such as ovarian cancer (29), renal cell carcinoma (31), lung adenocarcinoma (32), gastric carcinoma (33), and OS (34). Previous studies showed that SOX4 is regulated by miRNAs in OS and involved in the promotion of OS progression. For example, Liu et al (35) reported that miRNA-132 inhibits cell growth and metastasis in OS cell lines possibly by targeting SOX4. In line with previous findings, our results revealed that SOX4 was upregulated in OS tissues and cell lines, and SOX4 served as a direct target gene of miR-188. miR-188 expression was inversely correlated with SOX4 expression in OS tissues. Furthermore, the restoration of SOX4 reversed the effect of miR-188 on OS cells. Therefore, our findings demonstrated that miR-188 suppressed OS cell proliferation and invasion by targeting SOX4.

In conclusion, we revealed that miR-188 acted as a tumor suppressor by targeting SOX4 in OS. Our study provided novel insights into the design of therapeutic targets for OS intervention. 

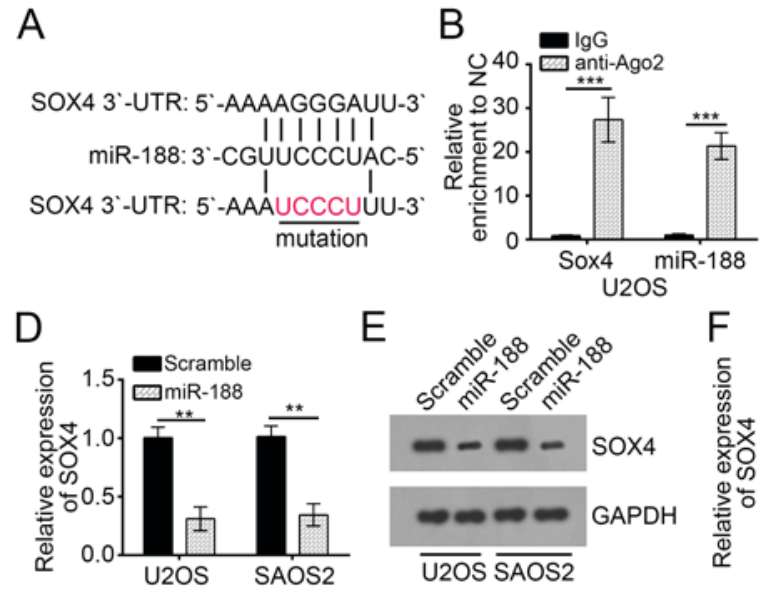

E

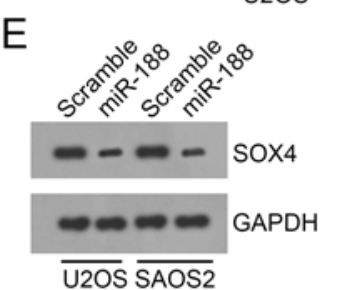

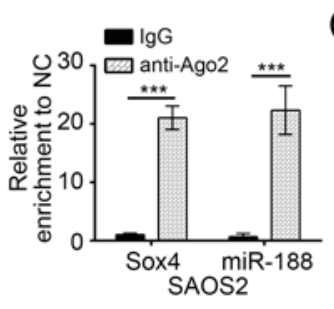

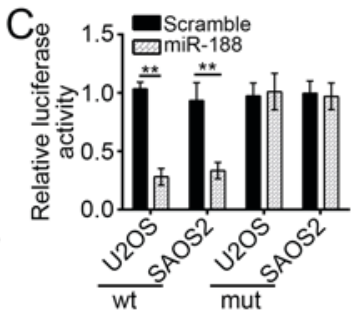

Figure 4. SOX4 is a direct target of miR-188 in OS cells. (A) Schematic of the miR-188 target sites in the 3'-UTR of SOX4 mRNA. (B) RNA immunoprecipitation assay with antibodies Ago2 or IgG from U2OS and SAOS2 cell extracts. RNA levels of SOX4 and miR-188 in immunoprecipitates were examined by RT-qPCR. (C) Luciferase activity of wild-type or mutant SOX4 3'-UTR reporter gene in U2OS and SAOS2 cells transfected with miR-188 mimics or control vectors. (D) mRNA and (E) protein levels of SOX4 were measured by RT-qPCR and western blot analysis in U2OS and SAOS2 cells. GAPDH served as the loading control. (F) RT-qPCR revealed that miR-188 expression was inversely correlated with that of SOX4 in OS tissues. Data are representative of three independent experiments and expressed as mean \pm standard deviation. ${ }^{* *} \mathrm{P}<0.01$ and ${ }^{* * * *} \mathrm{P}<0.001$ vs. the control group. OS, osteosarcoma; miR, microRNA; UTR, untranslated region; IgG, immunoglobulin G; NC, negative control; wt, wild-type; mut, mutant; RT-qPCR, reverse transcription-quantitative polymerase chain reaction.
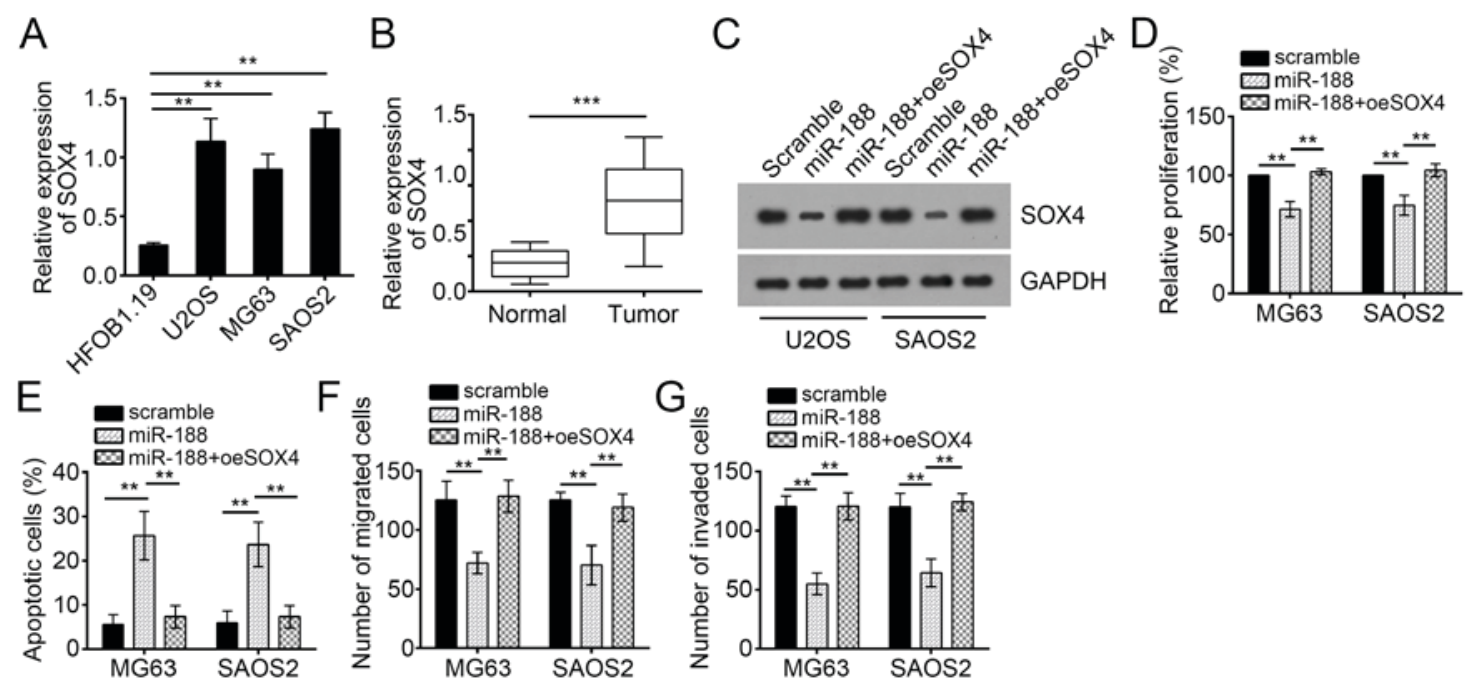

Figure 5. Ectopic SOX4 expression alleviates miR-188-mediated inhibition of the proliferation, migration and invasion of OS cells. RT-qPCR analysis of SOX4 expression in (A) OS cell lines and (B) OS tissues and adjacent normal tissues. (C) SOX4 protein levels were verified through western blot analysis in the indicated groups. (D) Cell proliferation, (E) apoptosis, (F) migration and (G) invasion were determined in the indicated cells through cell counting kit-8, fluorescence activated cell sorter and Transwell assays. Data are representative of three independent experiments and expressed as mean \pm standard deviation. ${ }^{* *} \mathrm{P}<0.01$ and ${ }^{* * *} \mathrm{P}<0.001$ vs. the control group. OS, osteosarcoma; miR, microRNA; RT-qPCR, reverse transcription-quantitative polymerase chain reaction.

\section{Acknowledgements}

Not applicable.

\section{Funding}

No funding was received.

\section{Availability of data and materials}

All data generated or analyzed during this study are included in this published article.

\section{Authors' contributions}

LP conceived and designed the study, interpreted the results and wrote the manuscript. LM, FL and LC performed the experiments. All authors read and approved the final manuscript.

\section{Ethics approval and consent to participate}

This study was approved by the Ethics Committee of the People's Hospital of Rizhao and all enrolled patients provided written informed consent. All procedures involving animals conformed 
to the national guidelines of and were approved by the Animal Care Ethics Committee of People's Hospital of Rizhao.

\section{Consent for publication}

Not applicable.

\section{Competing interests}

The authors declare that they have no competing interests.

\section{References}

1. Ottaviani G and Jaffe N: The epidemiology of osteosarcoma Cancer Treat Res 152: 3-13, 2009.

2. Mirabello L, Troisi RJ and Savage SA: Osteosarcoma incidence and survival rates from 1973 to 2004: Data from the surveillance, epidemiology and end results program. Cancer 115: 1531-1543, 2009.

3. Shen L, Wang P, Yang J and Li X: MicroRNA-217 regulates WASF3 expression and suppresses tumor growth and metastasis in osteosarcoma. PLoS One 9: e109138, 2014.

4. Ando K, Heymann MF, Stresing V, Mori K, Rédini F and Heymann D: Current therapeutic strategies and novel approaches in osteosarcoma. Cancers (Basel) 5: 591-616, 2013.

5. Leary SE, Wozniak AW, Billups CA, Wu J, McPherson V, Neel MD, Rao BN and Daw NC: Survival of pediatric patients after relapsed osteosarcoma: The St. Jude Children's Research Hospital experience. Cancer 119: 2645-2653, 2013.

6. Nana-Sinkam SP and Croce CM: Clinical applications for microRNAs in cancer. Clin Pharmacol Ther 93: 98-104, 2013.

7. Shen J, Stass SA and Jiang F: MicroRNAs as potential biomarkers in human solid tumors. Cancer Lett 329: 125-136, 2013.

8. Agrawal L, Sahu S, Ghosh S, Shiga T, Fujita D and Bandyopadhyay A: Inventing atomic resolution scanning dielectric microscopy to see a single protein complex operation live at resonance in a neuron without touching or adulterating the cell. J Integra Neurosci 15: 435-462, 2016

9. Ambros V: The functions of animal microRNAs. Nature 431: 350-355, 2004

10. Tan H, Zhu G, She L, Wei M, Wang Y, Pi L, Chen C, Zhang D, Tan P, Chen J, et al: MiR-98 inhibits malignant progression via targeting MTDH in squamous cell carcinoma of the head and neck. Am J Cancer Res 7: 2554-2565, 2017.

11. Xu FF, Xie WF, Zha GQ, Chen HW and Deng L: MiR-520f promotes cell aggressiveness by regulating fibroblast growth factor 16 in hepatocellular carcinoma. Oncotarget 8: 109546-109558, 2017.

12. Wang YN, Chen ZH and Chen WC: Novel circulating microRNAs expression profile in colon cancer: A pilot study. Eur J Med Res 22: 51, 2017

13. Fan Q, Hu X, Zhang H, Wang S, Zhang H, You C, Zhang CY, Liang $\mathrm{H}$, Chen $\mathrm{X}$ and $\mathrm{Ba} \mathrm{Y}$ : MiR-193a-3p is an important tumour suppressor in lung cancer and directly targets KRAS. Cell Physiol Biochem 44: 1311-1324, 2017.

14. Liu SY, Deng SY, He YB and Ni GX: miR-451 inhibits cell growth, migration and angiogenesis in human osteosarcoma via down-regulating IL 6R. Biochem Biophys Res Commun 482: 987-993, 2017.

15. Chen CZ: MicroRNAs as oncogenes and tumor suppressors. New Engl J Med 353: 1768-1771, 2005.
16. Ji X, Wang E and Tian F: MicroRNA-140 suppresses osteosarcoma tumor growth by enhancing anti-tumor immune response and blocking mTOR signaling. Biochem Biophys Res Commun 495: 1342-1348, 2018.

17. Dong C, Du Q, Wang Z, Wang Y, Wu S and Wang A: MicroRNA-665 suppressed the invasion and metastasis of osteosarcoma by directly inhibiting RAB23. Am J Transl Res 8: 4975-4981, 2016 .

18. Zhang H, Qi S, Zhang T, Wang A, Liu R, Guo J, Wang Y and $\mathrm{Xu}$ Y: miR-188-5p inhibits tumour growth and metastasis in prostate cancer by repressing LAPTM4B expression. Oncotarget 6: 6092-6104, 2015.

19. Livak KJ and Schmittgen TD: Analysis of relative gene expression data using real-time quantitative PCR and the 2(-Delta Delta C(T)) method. Methods 25: 402-408, 2001.

20. Wu X, Zhou H, Yue B, Li M, Liu F, Qiu C, Chen B and Ma X: Upregulation of microRNA-25-3p inhibits proliferation, migration and invasion of osteosarcoma cells in vitro by directly targeting SOX4. Mol Med Rep 16: 4293-4300, 2017.

21. Bao ZQ, Zhang CC, Xiao YZ, Zhou JS, Tao YS and Chai DM: Over-expression of Sox 4 and $\beta$-catenin is associated with a less favorable prognosis of osteosarcoma. J Huazhong Univ Sci Technolog Med Sci 36: 193-199, 2016.

22. Yu X and Li Z: Epigenetic deregulations in chordoma. Cell Prolif 48: 497-502, 2015.

23. Yu X and Li Z: The role of miRNAs in cutaneous squamous cell carcinoma. J Cell Mol Med 20: 3-9, 2016.

24. Lee H, Jee Y, Hong K, Hwang GS and Chun KH: MicroRNA-494, upregulated by tumor necrosis factor- $\alpha$, desensitizes insulin effect in C2C12 muscle cells. PLoS One 8: e83471, 2013.

25. Tian L, Guo Z, Wang H and Liu X: MicroRNA-635 inhibits the malignancy of osteosarcoma by inducing apoptosis. Mol Med Rep 16: 4829-4834, 2017.

26. Jia JP, Yin P, Han G, Xu M, Wang W and Bi WZ: MicroRNA-300 decreases cell viability, inhibits migration and promotes apoptosis of osteosarcoma cells via downregulation of Twist1. Mol Med Rep 16: 3613-3618, 2017.

27. Yang X, Cui Y, Yang F, Sun C and Gao X: MicroRNA-302a suppresses cell proliferation, migration and invasion in osteosarcoma by targeting ADAM9. Mol Med Rep 16: 3565-3572, 2017.

28. Wang L and Liu H: microRNA-188 is downregulated in oral squamous cell carcinoma and inhibits proliferation and invasion by targeting SIX1. Tumor Biol 37: 4105-4113, 2016.

29. Xi J, Feng J and Zeng S: Long noncoding RNA lncBRM facilitates the proliferation, migration and invasion of ovarian cancer cells via upregulation of Sox4. Am J Cancer Res 7: 2180-2189, 2017.

30. Ye X and Weinberg RA: Epithelial-mesenchymal plasticity: A central regulator of cancer progression. Trends Cell Biol 25: 675-686, 2015.

31. Tong Z, Meng X, Wang J and Wang L: MicroRNA-338-3p targets SOX 4 and inhibits cell proliferation and invasion of renal cell carcinoma. Exp Ther Med 14: 5200-5206, 2017.

32. Wang D, Gao ZM, Han LG, Xu F, Liu K and Shen Y: Long noncoding RNA CASC2 inhibits metastasis and epithelial to mesenchymal transition of lung adenocarcinoma via suppressing SOX4. Eur Rev Med Pharmacol Sci 21: 4584-4590, 2017.

33. Zhang M, Huang S and Long D: MiR-381 inhibits migration and invasion in human gastric carcinoma through downregulatedting SOX4. Oncol Lett 14: 3760-3766, 2017.

34. Wu X, Zhou H, Yue B, Li M, Liu F, Qiu C, Chen B and Ma X: Upregulation of microRNA-25-3p inhibits proliferation, migration and invasion of osteosarcoma cells in vitro by directly targeting SOX4. Mol Med Rep 16: 4293-4300, 2017.

35. Liu Y, Li Y, Liu J, Wu Y and Zhu Q: MicroRNA-132 inhibits cell growth and metastasis in osteosarcoma cell lines possibly by targeting Sox4. Int J Oncol 47: 1672-1684, 2015. 